\title{
Impact of Obesity on Outcomes of Patients With Hip Osteoarthritis Who Underwent Hip Arthroplasty
}

\author{
Hafeez Shaka ${ }^{1}$, Pius E. Ojemolon ${ }^{2}$ \\ 1. Internal Medicine, John H. Stroger, Jr. Hospital of Cook County, Chicago, USA 2. Anatomical Sciences, St. George's \\ University, St. George's, GRD
}

Corresponding author: Pius E. Ojemolon, piusojemolon@yahoo.com

\section{Abstract \\ Background}

While obesity has been clearly established as a risk factor for osteoarthritis (OA), there is a scarcity of studies comparing outcomes between obese and non-obese patients with hip OA who underwent hip arthroplasty.

\section{Methods}

This study involved adults with hip OA who had hip replacement procedures. Data was sourced from the Nationwide Inpatient Sample (NIS) database for 2016 and 2017. The primary outcome was inpatient mortality. Secondary outcomes included the development of non-ST segment elevation myocardial infarction (NSTEMI), sepsis, post-procedure site infection, pneumonia, acute kidney failure, deep vein thrombosis (DVT), pulmonary embolism, need for transfusion of blood products, complications involving orthopedic devices as well as mean length of hospitalization and mean total hospital charges.

\section{Results}

Obese patients did not have higher odds of in-hospital mortality (adjusted odds ratio [aOR]: 0.65, 95\% CI $0.303-1.381, \mathrm{p}=0.260)$, had increased mean length of hospitalization $(0.11,95 \% \mathrm{CI} 0.083-0.134, \mathrm{p}<0.001)$ and higher odds of developing DVT (aOR: 1.62, 95\% CI 1.187-2.222, p<0.001), acute kidney failure (aOR: 1.64, 95\% CI: 1.488-1.807, p<0.001) and pressure-related injuries (aOR: 1.64, 95\% CI 1.081-2.483, $\mathrm{p}=0.020$ ), compared with non-obese patients. Obese patients were found to have a lower aOR of having NSTEMI (aOR: $0.57,95 \%$ CI 0.332-0.986, $\mathrm{p}=0.044$ ), and need for blood product transfusion (aOR: $0.80,95 \%$ CI 0.726-0.875, $\mathrm{p}<0.001)$.

\section{Conclusion}

Although there is no difference in mortality among obese and non-obese patients who had hip arthroplasty, obese adults have increased odds of morbidity and perioperative complications. Hence, obese adults likely

Received 09/04/2020

Review began 09/09/2020 Review ended 10/03/2020 Published 10/10/2020

\section{(c) Copyright 2020}

Shaka et al. This is an open access article distributed under the terms of the Creative Commons Attribution License CC-BY 4.0., which permits unrestricted use, distribution, and reproduction in any medium, provided the original author and source are credited.
Categories: Endocrinology/Diabetes/Metabolism, Orthopedics, Rheumatology

Keywords: obesity, osteoarthritis, hip arthroplasty, surgical outcomes

\section{Introduction}

The World Health Organization defines obesity as abnormal or excessive fat accumulation that presents a risk to health. The most widespread statistical definition of obesity is a body mass index (BMI) of $30 \mathrm{~kg} / \mathrm{m}^{2}$ or more. The prevalence of obesity has markedly increased over the last three decades, and this is perpetuated by economic growth, mechanized transport, an increasingly sedentary lifestyle, and a nutritional transition to processed foods and high-calorie diets. It is estimated that by 2030, 20\% of the world's population will be obese [1]. Obesity is one of the most well-defined risk factors for osteoarthritis (OA).

Globally, OA is the most common degenerative disease of the joints, leading to substantial pain and disability world over [2]. After the knees and small joints of the hand, the hips are the most frequently affected joints in OA. Hip OA causes severe pain and stiffness of the hip joints leading to significant disability that often requires surgical intervention. Hip OA is the commonest reason for hip replacement surgery in the United States [3-6]. According to the Global Burden of Disease 2010 study, the global agestandardized prevalence of hip OA was $0.85 \%$ (95\% uncertainty interval 0.74\%-1.02\%) [7].

The association between obesity and OA has recently been shown to go beyond increased mechanical stress at joints. Systemic factors such as inflammatory mediators, cytokines, adipokines, growth factors, proteinases, and regulatory mechanisms (including interleukin-1, tumor necrosis factor-alpha [TNF- $\alpha$ ], 
interferon $\gamma$, transforming growth factor-beta [TGF- $\beta$ ], matrix metalloproteinases, and reactive oxygen species) have been implicated in the pathogenesis of OA. These systemic factors increase the susceptibility of joints to OA by direct damage to joint tissues or by impairing the process of repair in damaged joint tissues. These factors are also thought to be more relevant in the pathogenesis of hip and hand joint OA than knee OA [3,8-10].

While obesity has been clearly established as a risk factor for OA, there is a scarcity of studies comparing outcomes between obese and non-obese patients with hip OA who underwent hip replacement surgery. The primary aim of this study is to compare the inpatient mortality rates for patients with and without obesity admitted with hip OA who eventually underwent hip replacement using a nationally representative database. The secondary aim of the study is to investigate possible disparities in post-surgical complications between these two patient populations.

\section{Materials And Methods \\ Design and data source}

A retrospective cohort study was carried out among patients with hip OA who underwent hip arthroplasty during the same hospitalization. The Nationwide Inpatient Sample (NIS) database for 2016 and 2017 was used to obtain the study cohort. The NIS database is an administrative dataset containing discharge data to approximate national figures [11-12].

\section{Study population}

The study involved adults (aged 18 years and above) with a primary discharge diagnosis of hip OA who underwent any hip replacement procedure. Patients were excluded if they had revision arthroplasty, or if arthroplasty was done during a prior admission. This cohort was further divided based on the presence of at least one secondary discharge diagnosis of obesity, or a BMI greater than 30 .

\section{Outcome measures}

The primary outcome was inpatient mortality. Secondary outcomes included the odds of having a secondary discharge diagnosis of non-ST segment elevation myocardial infarction (NSTEMI), sepsis, post-procedure site infection, pneumonia, acute kidney failure, deep vein thrombosis (DVT), pulmonary embolism, need for transfusion of blood products, complications involving orthopedic devices as well as mean length of hospitalization and mean total hospital charges.

\section{Statistical analysis}

Data analysis was done using Stata ${ }^{\circledR}$ Version 16 software (StataCorp, TX, USA). Analyses were conducted using the weighting samples for national estimates in adjunct with Healthcare Cost and Utilization Project regulations for using the NIS database. A chi-square test was used to compare baseline characteristics and comorbidities between obese and nonobese groups. Outcomes were calculated using multivariate regression analysis to adjust for confounding variables (obtained from a literature review). A univariate screen was done to further confirm whether these factors affected outcomes with variables having a p-value less than 0.2 included in the multivariate regression analysis. The total length of hospital stay and the total hospital charges between the obese and non-obese groups were compared using a multivariate linear regression model. A p-value of $<0.05$ was set for statistical significance.

\section{Ethical considerations}

This study was exempt from Institutional Review Board approval as the database is de-identified and does not contain protected healthcare information.

\section{Results \\ Patient characteristics}

The combined NIS database for 2016 and 2017 contained over 71 million weighted hospital discharges of which 757,345 satisfied the inclusion criteria for the study. These patients were adults with a principal discharge diagnosis of hip OA who underwent hip replacement procedures. Of this group, just over a fifth (21.8\%) were obese, defined by International Classification of Diseases, 10th Revision (ICD-10) codes.

The obese patients were significantly younger (63.2 vs 66.2 years, $\mathrm{p}<0.001$ ), predominantly Caucasian females, and were majorly insured through Medicaid (48.6\%). Obese patients had more comorbidities including hypertension (62.2 vs 50.4\%, p<0.001), smoking history (36.4 vs 32.4\%, p<0.001), congestive heart failure (3.8 vs $2.1 \%, \mathrm{p}<0.001)$ and chronic kidney disease ( 5.8 vs $3.7 \%, \mathrm{p}<0.001)$ compared with non-obese patients. Patient and hospital characteristics are detailed in Table 1. 


\section{Cureus}

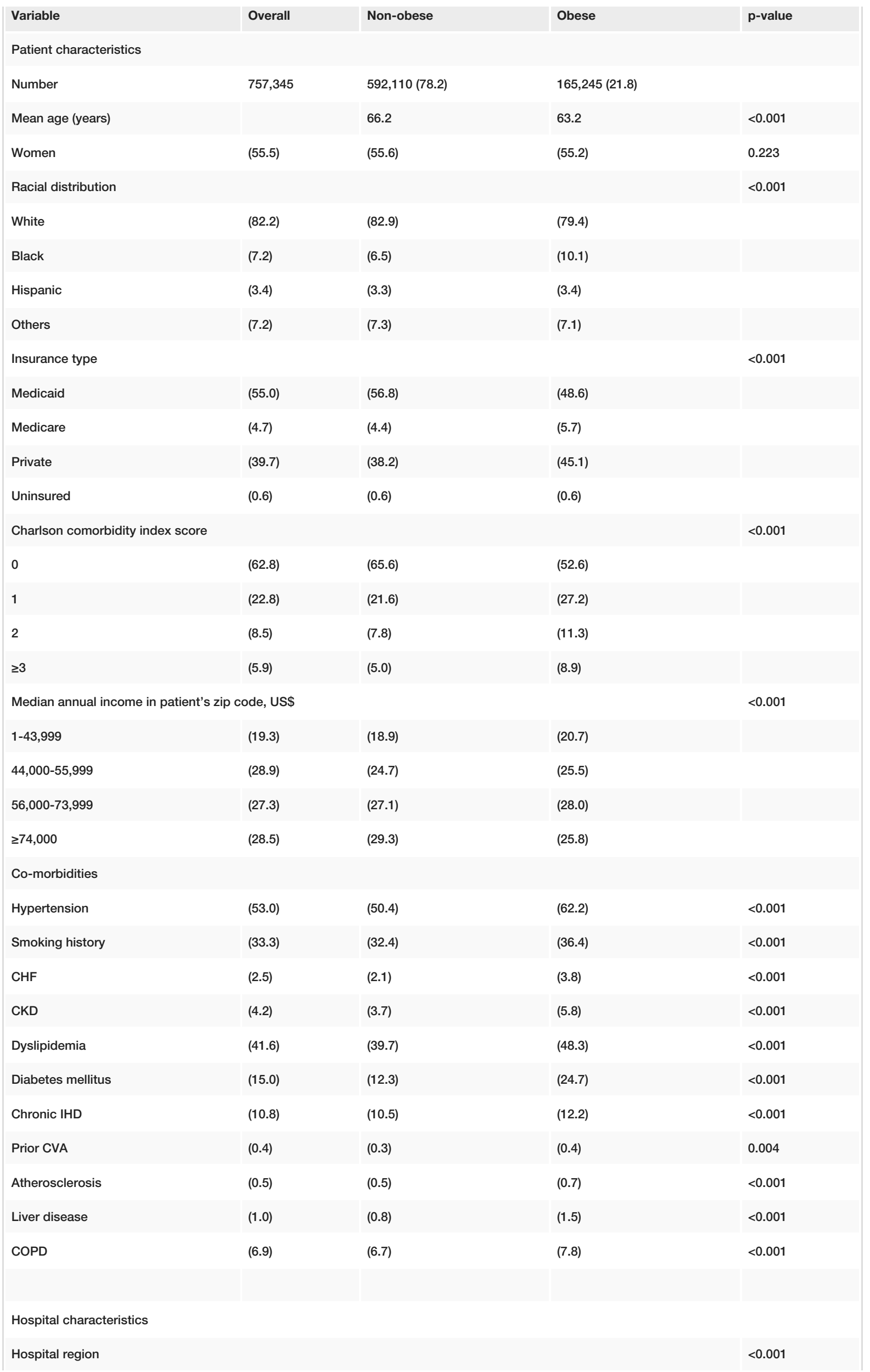




\section{Cureus}

\begin{tabular}{|c|c|c|c|c|}
\hline Northeast & (20.4) & (20.1) & (21.5) & \\
\hline Midwest & (26.1) & (24.9) & (30.3) & \\
\hline South & (32.4) & (33.1) & (29.8) & \\
\hline West & (21.1) & $(21.9)$ & (18.4) & \\
\hline Hospital bed size & & & & 0.1453 \\
\hline Small & (29.5) & (29.7) & $(29.0)$ & \\
\hline Medium & $(27.9)$ & $(28.1)$ & $(27.2)$ & \\
\hline Large & (42.6) & (42.2) & (43.8) & \\
\hline Urban location & (92.0) & (91.8) & (92.2) & 0.001 \\
\hline Teaching hospital & (64.8) & $(64.0)$ & (67.6) & $<0.001$ \\
\hline
\end{tabular}

\section{TABLE 1: Characteristics of patients with hip osteoarthritis who had hip replacement}

CHF: congestive heart failure; CKD: chronic kidney disease; CVA: cerebrovascular accident; IHD: ischemic heart disease; COPD: chronic obstructive pulmonary disease. Figures in parentheses are percentages.

\section{Primary outcome: in-hospital mortality}

The in-hospital mortality for patients with hip OA who had a replacement procedure was $0.032 \%$ of the total cohort. In total, there were 240 estimated deaths. Obese patients did not have higher odds of in-hospital mortality (adjusted odds ratio [aOR]: $0.65,95 \% \mathrm{CI} 0.303-1.381, \mathrm{p}=0.260$ ) when adjusted for co-morbidities.

\section{Secondary outcomes}

Obese patients had increased mean length of hospitalization $(0.11,95 \%$ CI $0.083-0.134, \mathrm{p}<0.001)$ compared to non-obese patients. There was, however, no difference in the mean total hospital charge between them. Obese patients were found to have a lower aOR of having a secondary discharge diagnosis of NSTEMI (aOR: $0.57,95 \%$ CI 0.332-0.986, $\mathrm{p}=0.044$ ), and need for blood product transfusion (aOR: 0.80, 95\% CI 0.726-0.875, $\mathrm{p}<0.001$ ). Obese patient, however, had higher odds of having a secondary discharge diagnosis of DVT (aOR: $1.62,95 \%$ CI 1.187-2.222, $\mathrm{p}<0.001$ ), acute kidney failure (aOR: 1.64, 95\% CI 1.488-1.807, $\mathrm{p}<0.001$ ), and pressure-related injuries (aOR: 1.64, 95\% CI 1.081-2.483, $\mathrm{p}=0.020$ ) compared to non-obese patients. Detailed outcomes are provided in Table 2. 


\section{Cureus}

\begin{tabular}{|c|c|c|c|c|}
\hline Outcome & Obese $(n=165,245)$ & Non-obese $(n=592,110)$ & Adjusted odds ratio $(95 \% \mathrm{Cl})$ & p-value \\
\hline \multicolumn{5}{|l|}{ Primary outcome } \\
\hline In-hospital mortality & 40 & 200 & $0.65(0.303-1.381)$ & 0.260 \\
\hline \multicolumn{5}{|l|}{ Secondary outcomes } \\
\hline Length of stay, mean days & 2.2 & 2.1 & $0.11^{\#}(0.083-0.134)$ & $<0.001^{*}$ \\
\hline Total hospital charges, mean US\$ & 60,750 & 60,551 & $-386^{\#}(-1341-568)$ & 0.428 \\
\hline NSTEMI & 85 & 465 & $0.57(0.332-0.986)$ & $0.044^{*}$ \\
\hline Sepsis & 150 & 320 & $1.34(0.831-2.131)$ & 0.235 \\
\hline Pneumonia & 275 & 825 & $1.02(0.738-1.400)$ & 0.919 \\
\hline Post-procedure infection & 45 & 105 & $1.34(0.538-3.359)$ & 0.527 \\
\hline Deep vein thrombosis & 305 & 750 & $1.62(1.187-2.222)$ & $0.002^{\star}$ \\
\hline Pulmonary embolism & 175 & 490 & $1.15(0.763-1.728)$ & 0.506 \\
\hline Complications from orthopedic device & 700 & 2480 & $0.97(0.805-1.180)$ & 0.793 \\
\hline Need for transfusion of blood products & 4610 & 19,950 & $0.80(0.726-0.875)$ & $<0.001^{*}$ \\
\hline Acute kidney failure & 4095 & 7900 & $1.64(1.488-1.807)$ & $<0.001^{\star}$ \\
\hline Pressure-related injury & 175 & 385 & $1.64(1.081-2.483)$ & $0.020^{*}$ \\
\hline
\end{tabular}

TABLE 2: Clinical outcomes in hospitalizations comparing non-obese versus obese patients principally admitted for hip osteoarthritis who had hip replacement surgery in the United States from 2016 to 2017

Cl: confidence interval; NSTEMI: non-ST segment elevation myocardial infarction.

*Statistically significant.

\#Mean difference.

\section{Discussion}

Obesity has been strongly associated with an increased risk of OA onset, faster progression, and more severe disability in patients with OA $[1,3,11]$. According to the Centers for Disease Control and Prevention, the ageadjusted prevalence of obesity (BMI $\geqslant 30 \mathrm{~kg} / \mathrm{m}^{2}$ ) was $42.4 \%$ among adults aged 20 and over in the United States in 2017-2018 [13]. This is almost twice as much as the prevalence of obesity in our study population (21.8\%) and may point towards deliberate attempts among OA patients to control their weight, as weight loss is the most commonly recommended lifestyle modification for obese patients with OA [14-16]. More than $55 \%$ of our study population were females, with similar representation in both the obese and non-obese cohorts. This is supported by several studies that highlight the increased risk of OA in females $[3,17,18]$.

According to our study, obesity was not shown to negatively affect in-hospital mortality rates among patients with hip OA who underwent hip replacement. Analysis of the patient characteristics in our study showed that obesity was associated with a significantly higher risk of co-morbidities such as hypertension, smoking history, congestive heart failure, chronic kidney disease, dyslipidemia,

atherosclerosis, liver disease, and chronic obstructive pulmonary disease, which on their own are associated with worse outcomes following hip replacement surgery [19]. After adjusting for these co-morbidities, there was no significant difference in the in-hospital mortality between both the obese and non-obese cohorts. There is a marked paucity of studies that highlight the effect of obesity on in-hospital mortality after hip replacement surgery. However, a population-based cohort study conducted by Tohidi et al., in which the authors followed up patients that had total hip arthroplasty for hip OA between 2002 and 2007, found that morbidly obese patients (BMI $>45 \mathrm{~kg} / \mathrm{m}^{2}$ ) were $38 \%$ more likely than non-morbidly obese patients to die within 10 years, after adjusting for baseline differences in age, sex, socioeconomic status, and comorbidity [20]. A similar study conducted by Ward et al. in 2015 found that patients who were morbidly obese were over twice as likely to die in the first year following total joint arthroplasty (hip or knee replacement) as non- 
morbidly obese patients (OR: 2.46, 95\% CI 1.34-4.52) [21]. The findings of increased mortality risk in the obese cohorts in these studies may however not directly be related to the effect of obesity on hip replacement surgery outcomes, but may be more in keeping with the increased risk of morbidity and mortality associated with obesity in the general population [22]. This highlights the need for more studies comparing in-hospital mortality between obese and non-obese patients who underwent hip replacement surgery for hip OA.

Our study also demonstrated significantly longer mean hospital length of stay, higher risk of NSTEMI, DVT, acute kidney injury, and pressure-related injuries in the obese cohort compared to non-obese patients. Similar findings were observed in a study conducted by Wallace et al. that showed that while an increased risk of DVT and longer hospital stays were observed among obese patients who had hip replacement surgery, no such association was observed for stroke and mortality [23]. These findings were also buttressed by the outcomes of a prospective cohort study conducted by Haebich et al. [24] and a systematic review conducted by Haynes et al. [25] that showed that obese patients are at an elevated risk of perioperative and postoperative complications when compared with non-obese controls. Additionally, our study showed no difference in mean hospital charges, risk of sepsis, pneumonia, post-procedure infection, pulmonary embolism, or complications from orthopedic devices. This is at variance with findings of a study conducted by Walls et al. that showed that following hip arthroplasty, morbid obesity was independently associated with superficial surgical site infection (OR: 2.02 with $95 \%$ CI 1.36-3.02, $\mathrm{p}<0.01$ ) and the composite endpoint of "any infection" (OR: 1.42 with 95\% CI 1.05-1.93, p<0.01) [26]. The effect of confounders like hypoalbuminemia that was significantly more in the obese cohort was however not ruled out and may explain the increased risk of infections in the obese cohort observed in the study.

Our study has several strengths. First, the data was sourced from the NIS, a large nationwide dataset, to provide a large sample size that enabled us to compare mortality outcomes despite the low inpatient mortality rate associated with this cohort of patients. Secondly, the nature of the database allows us to provide insights into the comparison of baseline demographics and hospital outcomes between hip OA hospitalizations with and without concomitant obesity to statistically significant levels.

There are some limitations to the study. First, NIS database studies are subject to non-randomization. Second, there may be coding errors, as the NIS is an administrative database that uses ICD-10 codes to characterize diagnoses and hospitalization events. Third, the NIS database deals with hospitalizations, not individual patients, so patients admitted multiple times will be counted multiple times. Fourth, there is no reliable way to determine if the secondary diagnoses preceded or developed during the index

hospitalization. Additionally, laboratory and radiologic data like erythrocyte sedimentation rate, C-reactive protein assay, and hip radiographs that could indicate underlying disease severity and inflammatory activity are not available in the NIS database.

\section{Conclusions}

Although there is no difference in mortality among obese and non-obese patients who had hip arthroplasty, obese adults have increased odds of morbidity and perioperative complications. Hence, obese adults likely require better perioperative management to decrease the incidence of these complications.

\section{Additional Information}

\section{Disclosures}

Human subjects: All authors have confirmed that this study did not involve human participants or tissue. Animal subjects: All authors have confirmed that this study did not involve animal subjects or tissue. Conflicts of interest: In compliance with the ICMJE uniform disclosure form, all authors declare the following: Payment/services info: All authors have declared that no financial support was received from any organization for the submitted work. Financial relationships: All authors have declared that they have no financial relationships at present or within the previous three years with any organizations that might have an interest in the submitted work. Other relationships: All authors have declared that there are no other relationships or activities that could appear to have influenced the submitted work.

\section{References}

1. Hruby A, Hu FB: The epidemiology of obesity: a big picture . Pharmacoeconomics. 2015, 33:673-689. 10.1007/s40273-014-0243-X

2. Chen D, Shen J, Zhao W, Wang T, Han L, Hamilton JL, Im HJ: Osteoarthritis: toward a comprehensive understanding of pathological mechanism. Bone Res. 2017, 5:16044. 10.1038/boneres.2016.44

3. Litwic A, Edwards MH, Dennison EM, Cooper C: Epidemiology and burden of osteoarthritis. Br Med Bull. 2013, 105:185-199. 10.1093/bmb/lds038

4. Hunter DJ, Bierma-Zeinstra S: Osteoarthritis. Lancet. 2019, 393:1745-1759. 10.1016/S0140-6736(19)304179

5. Varacallo M, Luo TD, Johanson NA: Total Hip Arthroplasty (THA) Techniques. StatPearls Publishing, Treasure Island, FL; 2020.

6. Ojemolon PE, Shaka H, Edigin E, et al.: Impact of diabetes mellitus on outcomes of patients with knee 
osteoarthritis who underwent knee arthroplasty: an analysis of the nationwide inpatient sample. Cureus. 2020, 12: e8902. 10.7759/cureus.8902

7. Cross M, Smith E, Hoy D, et al.: The global burden of hip and knee osteoarthritis: estimates from the Global Burden of Disease 2010 study. Ann Rheum Dis. 2014, 73:1323-1330. 10.1136/annrheumdis-2013-204763

8. Ashkavand Z, Malekinejad H, Vishwanath BS: The pathophysiology of osteoarthritis. J Pharm Res. 2013, 7:132-138. 10.1016/j.jopr.2013.01.008

9. Greene MA, Loeser RF: Aging-related inflammation in osteoarthritis. Osteoarthritis Cartilage. 2015, 23:1966-1971. 10.1016/j.joca.2015.01.008

10. Murphy NJ, Eyles JP, Hunter DJ: Hip osteoarthritis: etiopathogenesis and implications for management . Adv Ther. 2016, 33:1921-1946. 10.1007/s12325-016-0409-3

11. Healthcare Cost and Utilization Project - HCUP. Introduction to the HCUP National Inpatient Sample (NIS) . (2018). Accessed: May 14, 2020: https://www.hcup-us.ahrq.gov/db/nation/nis/NIS_Introduction_2016.jsp.

12. Houchens R, Ross D, Elixhauser A, Jiang J: HCUP Methods Series: Nationwide Inpatient Sample (NIS) Redesign Final Report - Report \#2014-04. Agency for Healthcare Research and Quality, Rockville, MD; 2014.

13. Hales CM, Carroll MD, Fryar CD, Ogden CL: Prevalence of obesity and severe obesity among adults: United States, 2017-2018. NCHS Data Brief. 2020, 360:1-8.

14. Bliddal H, Leeds A, Christensen R: Osteoarthritis, obesity and weight loss: evidence, hypotheses and horizons - a scoping review. Obes Rev. 2014, 15:578-586. 10.1111/obr.12173

15. Deveza L, Hunter D: An update on the treatment of osteoarthritis in obese patients . Expert Opin Pharmacother. 2016, 17:753-755. 10.1517/14656566.2016.1165208

16. Gersing AS, Schwaiger BJ, Nevitt MC, et al.: Is weight loss associated with less progression of changes in knee articular cartilage among obese and overweight patients as assessed with MR imaging over 48 months? Data from the Osteoarthritis Initiative. Radiology. 2017, 284:508-520. 10.1148/radiol.2017161005

17. Palazzo C, Nguyen C, Lefevre-Colau MM, Rannou F, Poiraudeau S: Risk factors and burden of osteoarthritis . Ann Phys Rehabil Med. 2016, 59:134-138. 10.1016/j.rehab.2016.01.006

18. Deshpande BR, Katz JN, Solomon DH, et al.: Number of persons with symptomatic knee osteoarthritis in the US: impact of race and ethnicity, age, sex, and obesity. Arthritis Care Res. 2016, 68:1743-1750. 10.1002/acr.22897

19. Schwartz FH, Lange J: Factors that affect outcome following total joint arthroplasty: a review of the recent literature. Curr Rev Musculoskelet Med. 2017, 10:346-355. 10.1007/s12178-017-9421-8

20. Tohidi M, Brogly SB, Lajkosz K, Harrison MM, Campbell AR, VanDenKerkhof E, Mann SM: Ten-year risk of complication and mortality after total hip arthroplasty in morbidly obese patients: a population study. Can J Surg. 2019, 62:442-449. 10.1503/cjs.017318

21. Ward DT, Metz LN, Horst PK, Kim HT, Kuo AC: Complications of morbid obesity in total joint arthroplasty: risk stratification based on BMI. J Arthroplasty. 2015, 30:42-46. 10.1016/j.arth.2015.03.045

22. Flegal KM, Kit BK, Orpana H, Graubard BI: Association of all-cause mortality with overweight and obesity using standard body mass index categories: a systematic review and meta-analysis. JAMA. 2013, 309:71-82. 10.1001/jama.2012.113905

23. Wallace G, Judge A, Prieto-Alhambra D, de Vries F, Arden N, Cooper C: The effect of body mass index on the risk of post-operative complications during the 6 months following total hip replacement or total knee replacement surgery. Osteoarthritis Cartilage. 2014, 22:918-927. 10.1016/j.joca.2014.04.013

24. Haebich SJ, Mark P, Khan RJK, Fick DP, Brownlie C, Wimhurst JA: The influence of obesity on hip pain, function, and satisfaction 10 years following total hip arthroplasty. J Arthroplasty. 2020, 35:818-823. 10.1016/j.arth.2019.09.046

25. Haynes J, Nam D, Barrack RL: Obesity in total hip arthroplasty: does it make a difference? . Bone Joint J. 2017, 99-B:31-36. 10.1302/0301-620x.99b1.bjj-2016-0346.r1

26. Walls JD, Abraham D, Nelson CL, Kamath AF, Elkassabany NM, Liu J: Hypoalbuminemia more than morbid obesity is an independent predictor of complications after total hip arthroplasty. J Arthroplasty. 2015, 30:2290-2295. 10.1016/j.arth.2015.06.003 